Check for updates

Cite this: RSC Adv., 2017, 7, 55957

\title{
Size-dependent optical properties of conjugated polymer nanoparticles
}

\author{
Ye Lin, ${ }^{a}$ Chaoging Dong, (D) ${ }^{\mathrm{b}}$ Fengwen Cao, ${ }^{a}$ Liqin Xiong, (D) ${ }^{\mathrm{a}}$ Hongchen $\mathrm{Gu}^{\mathrm{a}}$ \\ and Hong $X u$ (D) *a
}

A strong and stable fluorescent signal is the crux for the ultrasensitive biodetection technology, conjugated polymer nanoparticles (CPNs) as new fluorescence labels have attracted more and more attention for their excellent optical properties. However, a systematic understanding of the size-dependent optical properties of CPNs with diameters from the nano to submicron ranges is lacking, which is the most important issue when choosing label materials. Hence, poly[(9,9-dioctylfluorenyl-2,7-diyl)-co-(1,4-benzo- $\left\{2,1^{\prime}, 3\right\}$ thiadiazole)] (PFBT) nanoparticles with sizes from $50 \mathrm{~nm}$ to $200 \mathrm{~nm}$ were synthesized and studied. It was demonstrated that the spectroscopic and fluorescent properties are similar for CPNs with different sizes. The relationship between single-particle brightness and diameter was investigated via fluorescence spectrometry and fluorescence correlation spectroscopy (FCS), and the results presented that the singleparticle brightness increased quadratically with the increase of the diameter of CPNs. This research may provide valuable support to further application of CPNs in biological diagnostics.

Received 6th November 2017 Accepted 5th December 2017

DOI: 10.1039/c7ra12164d

rsc.li/rsc-advances labelling, ${ }^{11-13}$ in vivo imaging, ${ }^{14-18}$ biosensing, ${ }^{19}$ drug delivery ${ }^{20}$ and photodynamic cancer therapy. ${ }^{21}$

The preparation methods for CPNs include direct polymerization approach, ${ }^{22}$ miniemulsion technique, ${ }^{23}$ reprecipitation $\operatorname{method}^{24}$ and so forth. Among the various methods in preparing CPNs, post polymerization like miniemulsion and reprecipitation method are prevalent due to the facile and convenient synthetic process. Compared to miniemulsion method, reprecipitation method is easier to handle, has a higher production yield and could obtain brighter CPNs. ${ }^{25}$ Besides, it is worth noting that direct functionalization could be achieved in reprecipitation/coprecipitation synthetic process, without the need for extra complicated chemical modification. Hence, reprecipitation/coprecipitation techniques has become the most commonly adopted method to prepare CPNs to date. Zhang, X., et al. utilized direct functionalization methods to prepare the functionalized CPNs which was used in cellular labelling. ${ }^{26}$ In another study, Wu, C., et al. chose a kind of comblike amphiphilic polymer PS-PEG-COOH to coprecipitate with PFBT, obtaining ultrabright CPNs which were about 30 times brighter than IgG-Alexa 488 and Qdot $565 .^{11}$

However, in most cases, reprecipitation/coprecipitation techniques are adopted to synthesize CPNs in nano scale, followed by applying in in vivo imaging. For the in vitro detection of target biological molecules with ultra-low abundance like digital bio-detection, ${ }^{27}$ it is required that the fluorescent label has higher single-particle fluorescence intensity as well as a relatively small diameter for the sake of fast reaction kinetics. CPNs in the submicron range may become one of the most promising candidates for fluorescent labelling because they
${ }^{a}$ School of Biomedical Engineering/Med-X Research Institute, Shanghai Jiao Tong University, Shanghai 200030, P. R. China. E-mail: xuhong@sjtu.edu.cn

${ }^{b}$ School of Chemistry and Chemical Engineering, Shanghai Jiao Tong University, Shanghai 200240, P. R. China 
contain more chromophores than small nanoparticles and have relatively fast reaction kinetics in the meantime. Therefore, understanding the relationship between size and fluorescence properties of CPNs is the most basic issue to be addressed according to specific application requirements. Sun et $a .^{28}$ synthesized and studied the single particle fluorescence intensity of three CPNs composed of poly[2-methoxy-5-(2-ethylhexyloxy)-1,4-(1-cyanovinylene-1,4-phenylene)] (CN-PPV) with diameters of $16 \mathrm{~nm}, 33 \mathrm{~nm}$ and $59 \mathrm{~nm}$ which are usually called polymer dots (Pdots), ${ }^{\mathbf{1 0}}$ reaching a conclusion that there was a square dependence of single-particle brightness on particle size, whereas there is still a lack in a systematic and comprehensive research into the fluorescence properties of CPNs in submicron range, which is in urgent need for much wider application of CPNs in bio-detection. Therefore, in this study, PFBT CPNs ranging from $50 \mathrm{~nm}$ to $200 \mathrm{~nm}$ were synthesized firstly. Their absorption, excitation and emission spectra, relative and absolute quantum yield, together with single-particle brightness were studied in depth, providing detailed data and theoretical guidance for the better application of CPNs in biological assays.

\section{Experimental}

\section{Materials}

The conjugated polymer adopted in this study was poly[(9,9dioctylfluorenyl-2,7-diyl)-co-(1,4-benzo- $\left\{2,1^{\prime}, 3\right\}$-thiadiazole)]-Endcapped with DMP (PFBT, average molecular weight of 52000 , polydispersity of 3.4), which was purchased from American Dye Source, Inc. The amphiphilic comb like polymer, polystyrene graft ethylene oxide functionalized with carboxy (PS-PEG$\mathrm{COOH}$, total average molecular weight of 36500 , main chain average molecular weight of 6500, graft chain average molecular weight of 4600, polydispersity of 1.3), was purchased from Polymer Source Inc. Tetrahydrofuran (THF, SuperDry, 99.9\%) was purchased from J\&K Scientific Ltd. Millipore purified water $(18.2 \mathrm{M} \Omega \mathrm{cm})$ was used during the whole experiments. All chemicals were used without further purification and all experiments were performed at room temperature unless indicated otherwise.

\section{Synthesis methods}

The CPNs were synthesized via amphiphilic polymer coprecipitation method. First, THF was filtered with a $0.22 \mu \mathrm{m}$ syringe filter. PFBT and PS-PEG-COOH were dissolved in filtered THF to make a stock solution with a concentration of $40 \mathrm{mg} \mathrm{mL}$ respectively. Next, PFBT and PS-PEG-COOH solution were mixed and diluted with filtered THF, followed by sonication to form a homogeneous mixture with a given concentration. Then, the mixture was added quickly to $10 \mathrm{~mL}$ pure water and continuously sonicated for $3 \mathrm{~min}$. After that, the THF was removed by nitrogen purging on a $50{ }^{\circ} \mathrm{C}$ hotplate. Finally, the obtained suspension of nanoparticles was filtered with a $0.45 \mu \mathrm{m}$ syringe filter to gain a bottle of clear yellow liquid sample. CPNs with different diameters were synthesized through adjusting the concentration of PFBT, the temperature and the ratio of water in the diluted polymer mixture.

\section{Characterization methods}

Size, morphology and concentration measurement. The particle size and concentration were characterized by dynamic light scattering (DLS) and nanoparticle track analysis (NTA). Dynamic light scattering was performed on a Malvern Nano ZSP instrument. NTA was carried out on NanoSight LM10. During the NTA test, samples with proper concentration were injected into a detection cell, put onto the objective table, and adjusted to best focus. The motion of particles was recorded in a video and then the concentration and size of particles were calculated according to the motion trail and velocity of movement respectively. The microstructure and morphology of nanoparticles were observed by transmission electron microscopy (TEM). CPNs suspensions for TEM measurements were dropped onto copper grids and were dried at room temperature, and then the TEM images were obtained using a JEOL JEM-2100F transmission electron microscope.

Spectroscopic properties detection. UV-vis absorption spectra were recorded with an Aucy UV1901PC scanning spectrophotometer using $1 \mathrm{~cm}$ quartz cuvette. Fluorescence spectra were obtained using a Hitachi F-2700 fluorescence spectrophotometer.

Quantum yield measurement. Two different methods were adopted to measure the quantum yield (QY) of CPNs. For the test of relative QY, the QY of CPNs were compared with a fluorescent dye rhodamine 6G whose QY was known as 0.95. A diluted rhodamine $6 \mathrm{G}$ solution and CPNs suspension was first measured with UV/Vis spectrophotometer and the absorbance at $488 \mathrm{~nm}$ was recorded, making the absorbance lower than 0.05 to avoid significant self-absorption. Next, the same rhodamine 6G and CPNs sample was measured with fluorescence spectrophotometer (508-800 $\mathrm{nm}$ ) and the area of fluorescence peak was integrated. Then the QY of CPNs were calculated according to the following formula (eqn (1)), where the subscripts referred to the parameter of the corresponding substance.

Absolute QY of CPNs was measured via ZOLIX fluoroSENS9000 equipped with an integrating sphere. First, an empty integrating sphere was measured with exciting light $(375 \mathrm{~nm})$ and the initial spectrum (350-700 nm) was obtained. Second, the CPNs samples were put into the integrating sphere followed by excited directly with laser $(375 \mathrm{~nm})$, and the emission spectrum (350-700 nm) was recorded. Third, to measure the reabsorption/emission, the CPNs samples were still placed in the integrating sphere but not excited directly with laser, and the spectrum (350-700 nm) was recorded. Finally, the absolute QY of CPNs was given by the equation below (eqn (2)).

$$
\begin{aligned}
& \text { Relative } \mathrm{QY}_{\mathrm{CPNs}}= \mathrm{QY}_{\mathrm{dye}} \times \frac{\text { absorbance }_{\mathrm{dye}}}{\text { absorbance }_{\mathrm{CPNs}}} \\
& \times \frac{\text { emission integral }}{\mathrm{CPNs}} \\
& \times \frac{\text { emission integral }}{\mathrm{dye}} \\
& \text { refraction index } \text { index }_{\mathrm{C}_{2} \mathrm{H}_{5} \mathrm{OH}^{2}}
\end{aligned}
$$




$$
\text { Absolute QY }=\frac{P_{2}-(1-A) \times P_{3}}{A \times L_{1}}
$$

$L_{1}$ : the integrated value of excitation spectrum in the first step. $L_{2}$ : the integrated value of excitation spectrum after absorbed by CPNs in the second step. $L_{3}$ : the integrated value of excitation spectrum after re-absorbed by CPNs in the third step. $P_{2}$ : the integrated value of emission spectrum of CPNs in the second step. $P_{3}$ : the integrated value of re-emission spectrum of CPNs in the third step. $A=1-L_{2} / L_{3}$.

Single-particle brightness characterization. In this study, two different methods were adopted to characterize the single particle brightness of CPNs. The first one was to measure the fluorescence intensity of CPNs with known particle concentration, and single-particle brightness was given by the result of fluorescence intensity divided by particle concentration. The particle concentration of CPNs was characterized via NanoSight and the fluorescence intensity was obtained from fluorescence spectrophotometer. With the purpose of reducing the random error from experimental operation, the fluorescence intensity of CPNs with a series of concentration was measured, and the slope reflected the single-particle brightness of the synthesized PFBT CPNs.

The second method was to measure the single particle brightness of CPNs directly via fluorescence correlation spectroscopy (FCS). FCS is a novel class of single molecule detection technology through measuring the fluctuations of fluorescence intensity in a certain micro area due to Brownian motion or chemical reactions. ${ }^{29}$ Since the fluctuations are related to the concentration and chemical kinetic parameters of fluorescent molecules or particles, the information of a single molecule or particle could be obtained according to the standard equation for particles diffusing in a threedimensional Gaussian volume element (eqn (3)). ${ }^{30}$ The measurements were carried out with a home-built FCS system. ${ }^{31}$ The experiment process was as follows: the $488 \mathrm{~nm}$ laser beam (argon ion laser, from Ion Laser Technology, Shanghai, China) was adjusted to $c a .133 \mathrm{nW}$ through a circular neutral density filter, then enlarged through a telescope to exactly fill the back aperture of the objective lens. Next, the enlarged light spot was focused by a water immersion objective (UplanApo, 60 NA1.2, Olympus, Japan) into the sample. Before the laser entering the objective, the excitation intensity was recorded via an optical power meter (HIOKI 3664, Shanghai, China). While the excited fluorescence signal was collected by the same objective and went through a dichroic mirror (505DRLP, Omega Optical, USA). Then the fluorescence was coupled into a $35 \mathrm{~mm}$-pinhole at the image plane ahead the single-photon counting module (SPCM-AQR16, Perkin Elmer EG\&G, Canada) where the fluorescence intensity was measured. Finally, the fluorescence fluctuations were correlated by a digital correlator card (http://www.correlator.com, Shenzhen, China). The acquired raw FCS data were analysed with eqn (3). The parameters concerning the condition of the detection region can be obtained by fitting the autocorrelation curve of Rhogreen with eqn (3), whose diffusion coefficient in water is $2.8 \times 10^{-6} \mathrm{~cm}^{2} \mathrm{~s}^{-1}$ at $25^{\circ} \mathrm{C}$. For fluorescent materials with almost no triplet states like PFBT CPNs, the average number of emitted fluorescent molecules in the detection volume could be easily obtained as it is equal to the inverse $G(0)$. Brightness per particle (BPP) could be calculated from the particle number $(N)$ and the recorded fluorescence intensity.

$$
\begin{aligned}
G(\tau)= & \left(1+\frac{\left.T \times \mathrm{e}^{-\tau / \tau_{\text {triplet }}}\right) \frac{1}{N} \times \frac{1}{\left(1+\frac{\tau}{\tau_{\mathrm{D}}}\right)}}{} \times \frac{1}{\sqrt{1+\left(\frac{\omega_{0}}{z_{0}}\right)^{2} \times \frac{\tau}{\tau_{\mathrm{D}}}}}\right.
\end{aligned}
$$

$T$ : the fraction of fluorescent molecules in the triplet state. $N$ : the average number of emitted fluorescent molecules in the detection volume. $\tau_{\text {triplet }}$ : the lifetime of the triplet state. $\tau_{\mathrm{D}}$ : the characteristic diffusion time of fluorescent molecules in the detection volume. $\omega_{0}$ and $Z_{0}$ : the lateral radius and axial radius of the detection volume.

\section{Results and discussion}

\section{Conjugated polymer nanoparticle preparation and size control}

In this study, amphiphilic polymer coprecipitation method was adopted to synthesize CPNs. The detailed synthetic procedure of CPNs is presented in Scheme 1. First, PFBT and PS-PEG$\mathrm{COOH}$ were dissolved in THF. Then the mixture of the two polymers were added quickly into deionized water and continuously sonicated for $3 \mathrm{~min}$. After that, THF was removed by nitrogen sweeping. Since PFBT and PS are both hydrophobic, their chains tend to assemble and aggregate to form particles in watery environment based on hydrophobic interaction. While the hydrophilic PEG chains are inclined to stay on the surface to stabilize the synthesized nanoparticles. At last, the above mixture was filtered and the brilliant yellow CPNs suspension was successfully obtained.

The conjugated polymer employed in this study was PFBT, whose structural formula is shown in Fig. 1a. The conjugated structure of biphenyl and benzothiophene enables electrons to move along the polymer chain, exhibiting a direct band gap which leads to an efficient absorption or emission at the band gap. ${ }^{10}$ The amphiphilic polymer adopted was a come-like copolymer composed of hydrophobic PS as the main chain and hydrophilic PEG as the branched chains. PS could entangle with PFBT through hydrophobic interaction, while PEG plays an important role in keeping the nanoparticles dispersing stably in water. The inset images in Fig. 1a show the aqueous suspensions of the resulted PFBT nanoparticles under room light and UV light respectively. The as-synthesized CPNs suspension exhibited light yellow under room light, and emitted brilliant yellow fluorescence under UV light (365 nm), revealing a strong fluorescence from CPNs.

Here, CPNs with a series of particle sizes were controllably synthesized via changing the experimental conditions which will be mentioned later. A typical TEM picture of CPNs (Fig. 1b) 


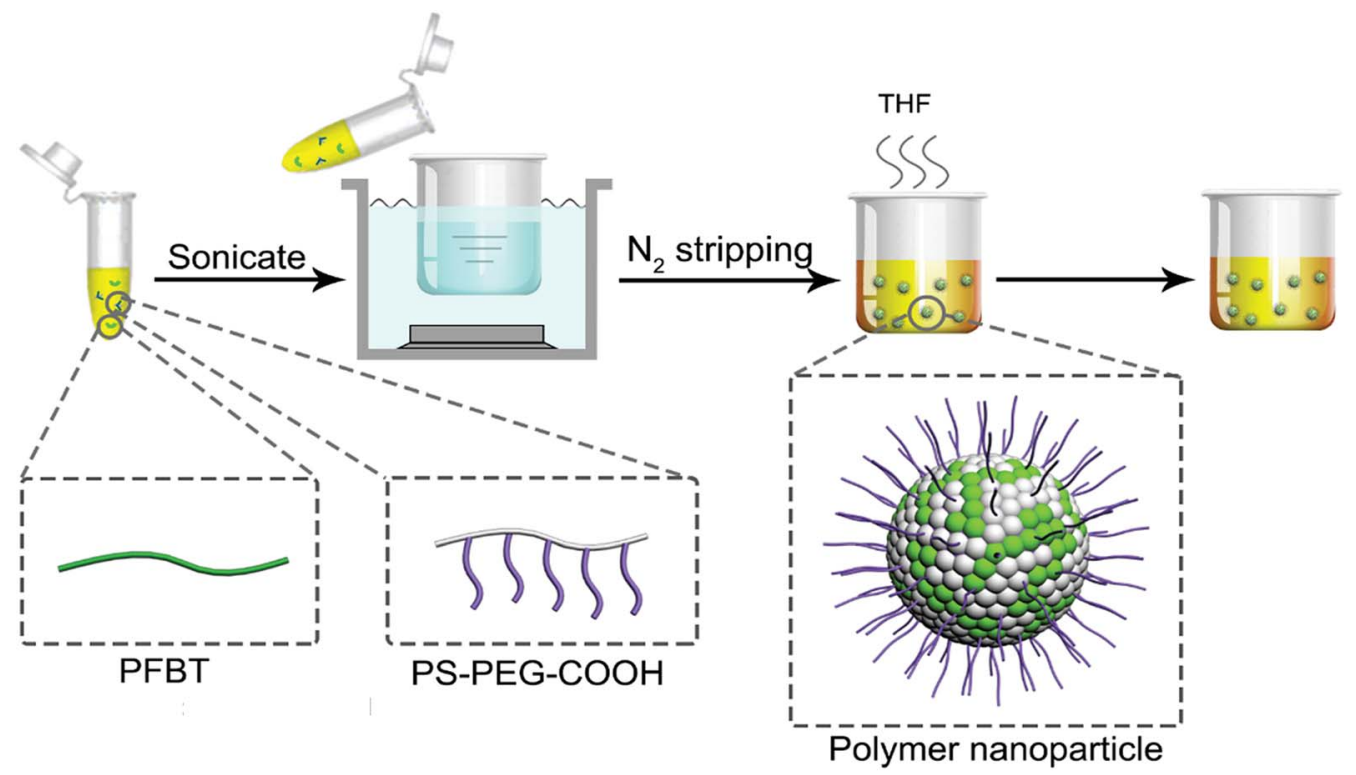

Scheme 1 Schematic showing of the synthesis of CPNs.

(a)

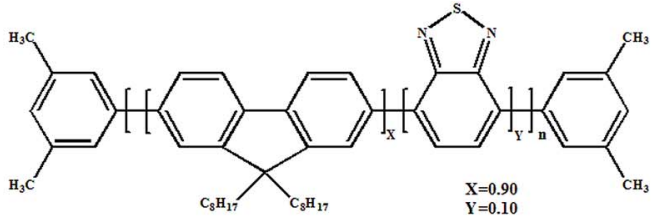

(b)
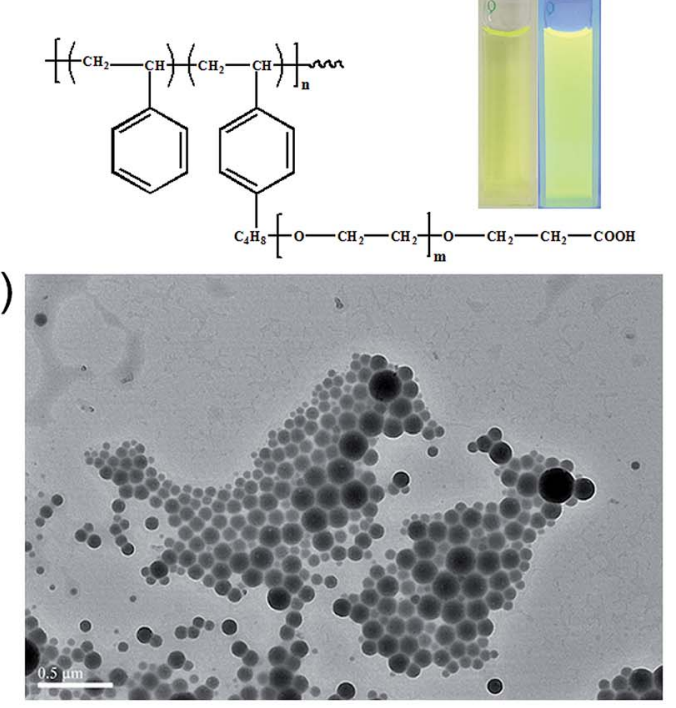

(c)

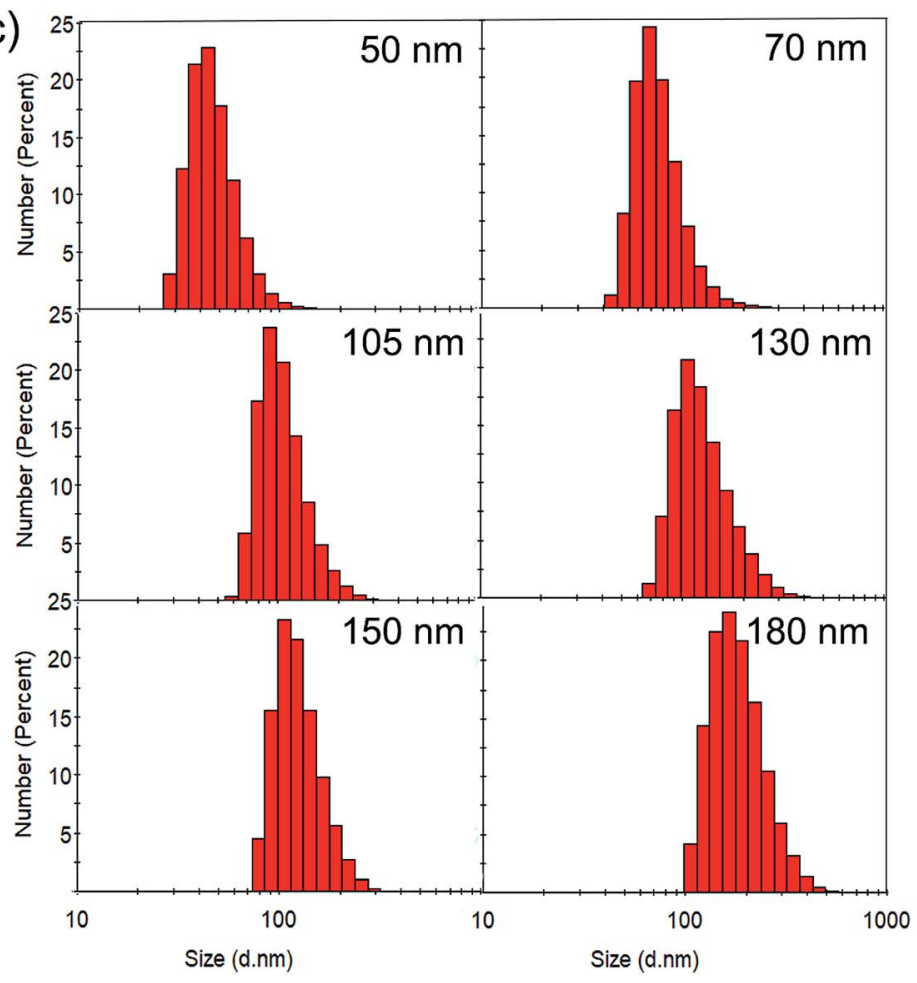

Fig. 1 (a) Chemical structure of semiconductor polymer PFBT and PS-PEG-COOH. The inset shows aqueous suspensions of PFBT nanoparticles under room light and UV light respectively. (b) TEM image of PFBT nanoparticles with a DLS diameter (number average) of $167 \mathrm{~nm}$. (c) DLS size distributions of PFBT nanoparticles with different diameters.

shows that the synthesized nanoparticles were in perfect spherical shape with a certain size distribution. The representative number average particle size and size distribution of CPNs are exhibited in Fig. 1c and Table 1. By means of changing the synthesis condition, the diameter of resulted CPNs ranged from $50 \mathrm{~nm}$ to $200 \mathrm{~nm}$. Otherwise, according to the measuring results of CPNs from DLS, the PDI of size distribution index were around 0.1 (Table 1). The negative zeta potential of approximately $-25 \mathrm{mV}$ (Table 1) illustrates that PS-PEG-COOH was successfully integrated into the as-synthesized CPNs. 
Table 1 The number-average diameter, PDI and zeta potential of the synthesized CPNs measured by DLS

\begin{tabular}{|c|c|c|c|c|c|c|c|c|c|c|c|c|c|}
\hline Number-average diameter (nm) & 50 & 60 & 70 & 80 & 90 & 105 & 115 & 125 & 140 & 152 & 167 & 183 & 200 \\
\hline PDI & 0.094 & 0.168 & 0.111 & 0.090 & 0.073 & 0.086 & 0.062 & 0.065 & 0.182 & 0.063 & 0.064 & 0.089 & 0.037 \\
\hline Zeta potential $(\mathrm{mV})$ & -22.0 & -26.8 & -25.6 & -18.0 & -16.6 & -15.8 & -28.7 & -31.8 & -22.5 & -27.9 & -23.5 & -33.9 & -23.3 \\
\hline
\end{tabular}

To achieve the controllability towards the size of the resulted CPNs, in this research, the influence of the concentration of PFBT, the quality of solvent in the polymer mixture, and the temperature of sonication water on the size of CPNs were carefully studied respectively. Fig. 2 demonstrates the relationship between the three factors and the diameter of CPNs. The diameter mentioned in this study refers to the number average diameter measured by DLS unless specified. As shown in Fig. 2a, the diameter of CPNs had a significant increase when the concentration of polymer increased. The effect can be explained from in-depth understanding of the nanoparticle formation process. At first, PFBT and PS-PEG-COOH were both well dissolved in THF, with stretched chain segments. When the two components were added into water which is the poor solvent for PFBT and PS-PEG-COOH, PFBT and PS chains tended to tangle each other based on their hydrophobic interaction and a kind of dense polymer aggregation formed quickly, while PEG preferred to stay onto the interface to stabilize the nanoparticle. In polymer solution of higher concentration, there were more chain segments in a given volume, leading to more chain tangling together to form CPNs with larger size as was expected. In addition, as shown in Fig. $2 \mathrm{~b}$, with the increase of ultrasonic water temperature, the size of CPNs also enlarged obviously. The outcome is consistent with a previous study, although the adopted conjugated polymers are different. ${ }^{32}$ This is because the thermal motion of chain segments become more violent as the temperature rises, increasing the chances of chain entanglement, therefore, the diameter of the synthesized CPNs increases. Furthermore, as shown in Fig. 2c, the water content in polymer solution appeared to have a complicated impact on the size of CPNs. Increase of water content not only resulted in increasing the diameter of CPNs but may also lead to a wider size distribution. When water content was $30 \%$, there was a dramatic increase in size of the obtained CPNs. This may be resulted from the poor solubility of conjugated polymers in mixed solvent. Since water is the poor solvent of the two polymers, the extensibility and locomotivity of PFBT chains are constrained when adding water into the reaction system, causing larger CPNs. In addition, the extensibility and locomotivity of PFBT chains are different in various microenvironments, thus resulting in different bending ability when PFBT was transferred from good solvent into poor solvent, finally leading to a wider size distribution of CPNs. This also increased the uncertainty in the preparing process, possibly resulting in a huge difference in the mean diameter of CPNs with a wide distribution, which was reflected in the size decrease when the water content increased to $50 \%$. Besides, the impact of the ratio between the two polymers, the dosage of PFBT, the sonication power and time were studied but not presented here, since the size of CPNs didn't have significant difference with the change of these factors, indicating that these factors had neglected effect on the conformation of CPNs.

\section{Analysis on the spectroscopy of PFBT CPNs}

As the excellent fluorescent properties are crucial to the wide application of CPNs, grasping the rules of CPNs in optical phenomena is of great importance. The spectra are able to reflect the fundamental photophysical properties of CPNs, therefore measuring the absorption, excitation and emission spectra of CPNs is an indispensable task to deeply investigate into CPNs. The spectroscopic characteristics of the synthesized PFBT CPNs with various diameters are shown in Fig. 3. First, Fig. 3a demonstrates the absorption spectrum of original PFBT and obtained CPNs with different sizes. PFBT dissolved in THF had an absorption spectrum with a peak at $380 \mathrm{~nm}$ and a shoulder peak at $440 \mathrm{~nm}$. After nanoparticle formation, the absorption peak wavelengths of the nanoparticles red shifted from $378 \mathrm{~nm}$ to $399 \mathrm{~nm}$ with the increasing of particle size.
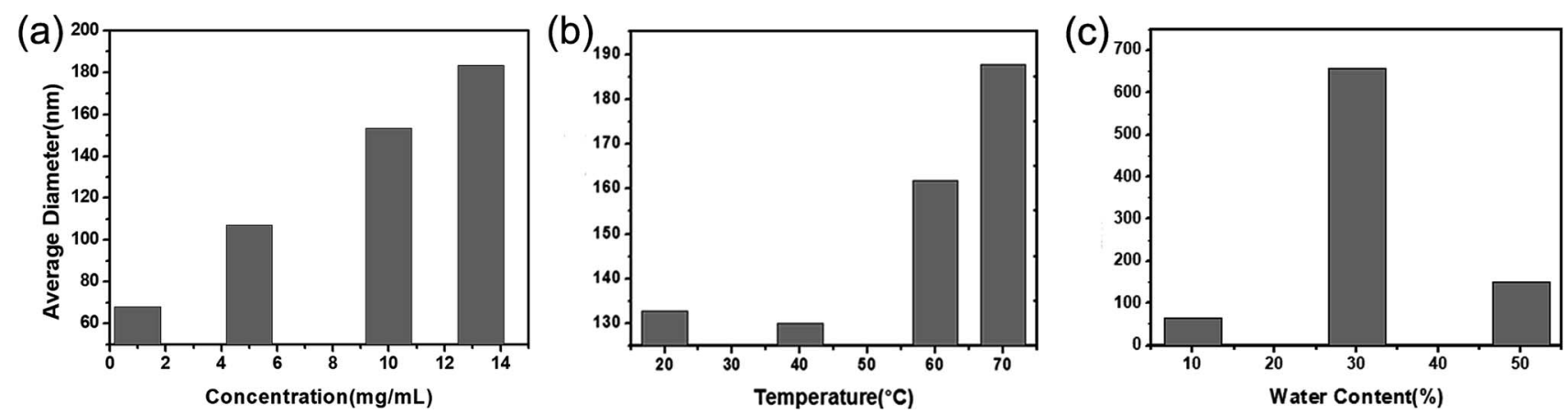

Fig. 2 (a) The relationship between the concentration of PFBT and the average diameter of obtained PFBT nanoparticles. (b) The relationship between temperature and the diameter of obtained PFBT nanoparticles. (c) The relationship between the water content in solvent and the diameter of obtained PFBT nanoparticles. 
(a)

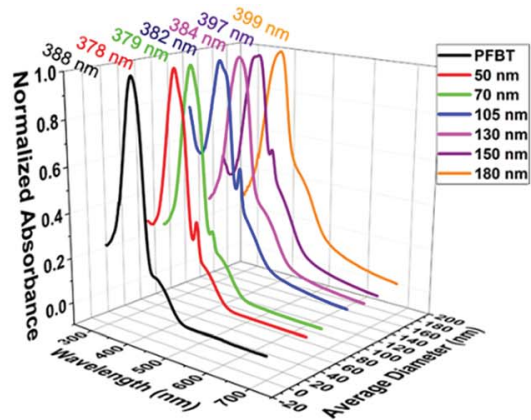

(c)

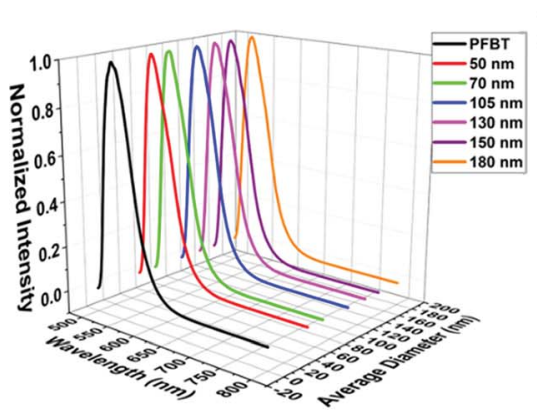

(b)

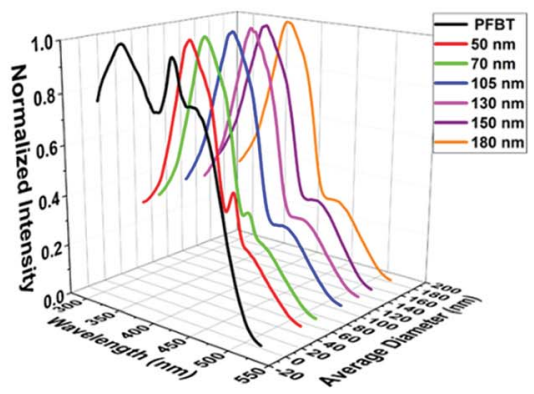

(d)

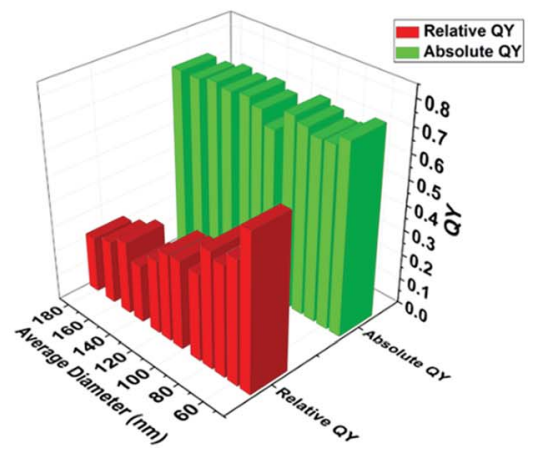

Fig. 3 (a) Absorption spectra, (b) excitation spectra, (c) emission spectra and (d) relative and absolute quantum yield of PFBT nanoparticles with different diameters.

This phenomenon maybe results from the fluorescence mechanism of CPNs reported previously. ${ }^{33}$ Due to some polymer chain segments are in good packing in certain regions of CPNs, where the conjugated chain lengths are longer and the interchain electron interactions stronger, leading to lower energy in these regions (so called red sites ${ }^{33}$ ). While those conjugated chain lengths in the majority of CPNs are shorter, where the energy are higher and are called blue sites. Every chromophore could absorb photons and form excitons, but excitons in blue sites would quickly transfer to red sites. The energy transfer leads to the fact that the emission of CPNs mainly originates from singlet excitons in red sites, while the absorption derives from wider spread blue sites. So, the spectra properties should closely relate to the inner chain conformation of CPNs. However, for nanoparticles, the absorbance obtained from ultraviolet spectrophotometer includes not only absorption from chromophores but also light scattering from the nanoparticle itself. It has been found that light scattering makes an increasingly important contribution to the extinction with the increasing of the nanoparticle size. ${ }^{34}$ Therefore, the difference in polymer chain structure and light scattering behaviour of obtained CPNs may both contribute to the red shift of the absorption peak. It is presented in Fig. 3a that, as the size of CPNs increases from $50 \mathrm{~nm}$ to $200 \mathrm{~nm}$, the absorbance of CPNs at $700 \mathrm{~nm}$ increases accordingly from 0.0003 to 0.005 , showing a more than 10 -fold increase, which indicated that the absorption intensity from particle's light scattering behaviour was making a nonignorable contribution to the detected absorbance.

As we known, the absorption spectra represent the absorbance of a certain substance at different wavelength. While the excitation spectra exhibit the fluorescence intensity of a fluorescent material at a given emission wavelength under different excitation wavelength, reflecting the influence of different excitation wavelength on fluorescence intensity. Because the scattering portion of exciting light will not make contributions to fluorescence, the excitation spectra could reflect the true absorption property for materials with low quantum yield of triplet to some degree. Owing to the uncertainty of the scattering proportion in the absorbance tested via ultraviolet spectrophotometer, the true absorption spectra of CPNs is inaccessible. Hence, the excitation spectra become ideal choice to verify whether the red shift in absorption spectra roots in the difference in the conformation of CPNs or not. Compared to PFBT dissolved in THF, the peak wavelengths of CPNs in excitation spectrum had an apparent red shift (from $339 \mathrm{~nm}$ to 370 $\mathrm{nm}$ ), as demonstrated in Fig. 3b. For PFBT dissolved in THF, the polymer chains are in random coil conformation and the conjugated chain length is relatively short, so the corresponding $\pi-\pi^{*}$ energy gaps are high, leading to short peak wavelength. For CPNs, the polymer chains suffered from a quality decrease in solvent, and some polymer chain segments tend to arrange orderly resulting in longer conjugated chain length, the peak wavelength red shifted afterwards. Nevertheless, unlike the absorption spectra, the excitation spectra of nanoparticles with different sizes had little difference. They were almost the same in both the peak shape and position. To a large extent, this result manifests that the red shift in absorption spectra as the diameter of CPNs increases mainly originates from light scattering of nanoparticles. In the meantime, it indicates clearly that CPNs with different sizes have similar inner chain structure and length. 
Fig. 3c presents the fluorescence spectra of PFBT solution in THF and CPNs in water. In comparison to PFBT dissolved in THF, the peak wavelengths of CPNs in fluorescence spectra had a little blue shift. After nanoparticle formation, the PS chains replaced THF to surround the PFBT chains. The lower polarity of PS chains may be the origin of the slight blue shift in the fluorescence spectra of CPNs compared to PFBT dissolved in THF. Whereas, resembling to excitation spectra, the fluorescence spectrum of nanoparticles with different sizes had little difference, further conforming the speculation that nanoparticles with various sizes have similar chain structure and length. Different from what we found above, Kurokawa and coworkers $^{32}$ discovered that the absorption and emission spectra of P3DDUT CPNs red shifted as the diameter increased. This discrepancy maybe results from the different conformation of CPNs derived from different species of conjugated polymers.

Another important parameter reflecting the fluorescence property of CPNs is quantum yield (QY). Here, the relative QY was obtained by referring to rhodamine 6G (fluorescent dye with known QY of 0.95). While integrating sphere instrument was employed to collect the whole excitation and emission light in the detection of absolute QY.

The relative and absolute QY of CPNs with various sizes are exhibited in Fig. 3d. With the increase of the diameter of CPNs, their absolute QY did not change much and were approximately 0.7 . It is indicated that the efficiency of electrons transferring from blue sites to red sites is almost the same with the change of size. This also illustrates that CPNs could maintain excellent optical properties as its size grows. On the contrary, the relative QY of CPNs had a declining trend when its diameter increased. The interesting discrepancy between relative and absolute QY may ascribe to the difference in the measuring process. In the detection of absolute QY, the employment of integrating sphere were able to collect the whole absorption light. Nonetheless, in the measurement of relative QY, the number of absorbed photons was represented by the absorbance tested via ultraviolet spectrophotometer. As mentioned previously, the absorbance measured via ultraviolet spectrophotometer includes the absorption from chromophores and light scattering from the nanoparticle itself. With the increasing of diameter, the proportion of scattering in the total absorbance increases significantly, so the absorbance gets further and further deviated from the true absorption, leading to a gradual decline in the calculated relative QY.

\section{Size-dependent single-particle fluorescent brightness}

With regard to the fluorescent label technology, single-particle fluorescence intensity is one of the most important index which directly relates to the bio-detection performance to draw the most attention, like the limit of detection or detection linear range. Hence, having a clear knowledge of the size-dependent single-particle fluorescent brightness of CPNs provides significant support in its application.

In this study, two different methods were adopted to characterize the single particle fluorescent brightness of
CPNs. The first one was to measure the fluorescence intensity of CPNs with known particle concentration, single-particle fluorescent brightness was given by the result of fluorescence intensity divided by particle concentration. As demonstrated in Fig. 4a, the fluorescence intensity of per ten billion nanoparticles increased with the increasing of diameter. Furthermore, as illustrated in Fig. 4c, it was worked out that the single particle fluorescent brightness of CPNs had an approximately quadratic increase as the diameter increased. This result was in consistency with the findings of Sun et al. ${ }^{28}$ However, according to above results that the QY of CPNs maintained almost the same as size varied, it takes for granted to speculate that the single particle fluorescence intensity of CPNs should be proportional to the cube of diameter theoretically. The unexpected experiment result may come from the deviation of the hydrodynamic diameter tested by DLS, which was usually larger than the true diameter of CPNs. Besides, the uneven distribution of particle size may also contribute to the difference between theoretical calculations and testing results.

The second method was to measure the single particle fluorescent brightness of CPNs directly via fluorescence correlation spectroscopy (FCS). ${ }^{35}$ FCS is a novel class of single molecule detection technology, which acquires the dynamic parameters and microscopic information of nanoparticles through measuring the fluctuations of fluorescence intensity in a certain micro area. The fluorescence auto-correlation function is a good approximation for molecules undergoing free diffusion through a confocal detection volume. According to the fluorescence auto-correlation function, the particle number $(N)$ in the micro area could be obtained. The single particle brightness is calculated as the fluorescence intensity divided by particle number. According to Fig. 4b, the result from FCS was that the single particle fluorescent brightness increased as diameter increased from $50 \mathrm{~nm}$ to $200 \mathrm{~nm}$, which was in accordance with that from the first method. It was also verified in Fig. 4d that there was an approximative square dependence between single particle brightness and the diameter of CPNs.

To have a clearer understanding how bright the CPNs are, comparison of the single-particle fluorescent brightness between the resulted CPNs and FITC-doped silica spheres with similar size which was another species of high-bright nanoparticles known as C-dots ${ }^{36}$ were performed by means of the first method. As illustrated in Table 2, the fluorescence intensity slope of PFBT nanoparticles was approximately 20 times higher than that of FITC-doped silica nanoparticles, indicating that the prepared PFBT nanoparticles were ultra-bright fluorescent materials.

It should be noted that all the test results in this work are statistical results, so this study reveals the correlation between average diameter and "average optical properties" of CPNs. On the basis of the above findings, so far, it's more clear and convenient to accurately choose CPNs with suitable size according to the required fluorescence intensity, facilitating further application of CPNs in biodetection. 
(a)

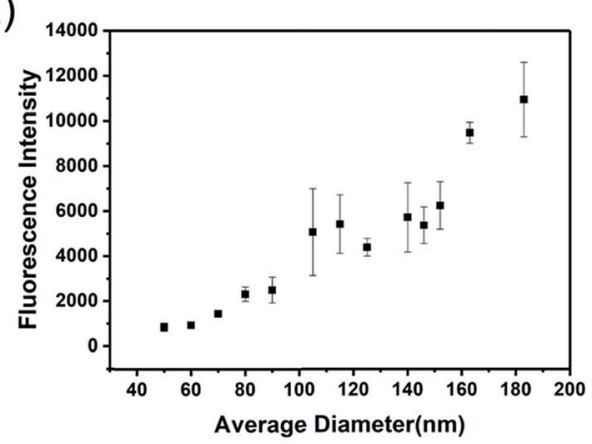

(c)

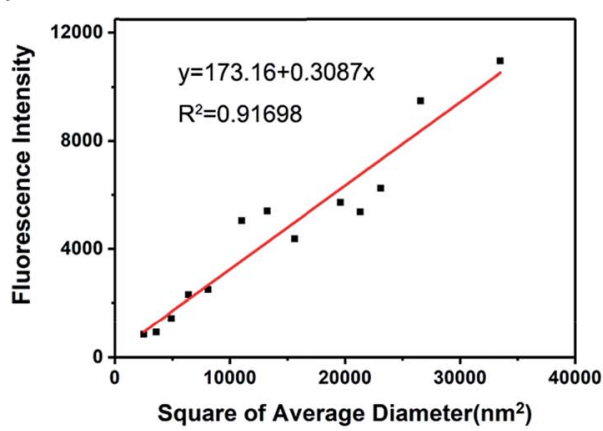

(b)

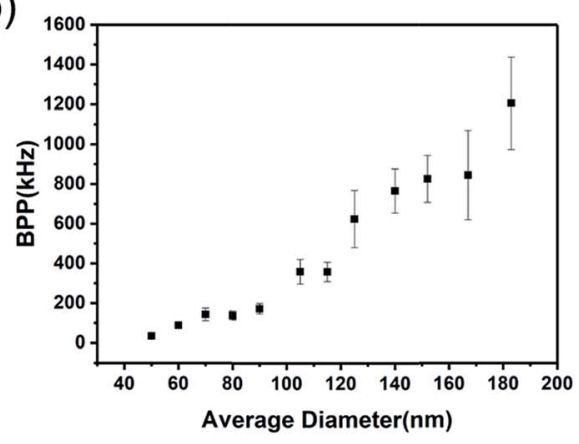

(d)

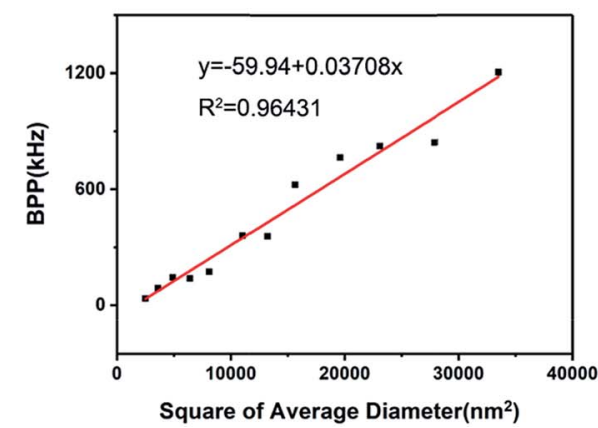

Fig. 4 Single particle fluorescence intensity of PFBT nanoparticles with different DLS diameters characterized by (a) NanoSight and fluorescence spectrophotometer, and (b) fluorescence correlation spectroscopy (FCS). Linear fitting of single particle fluorescence intensity of PFBT nanoparticles with square of diameters characterized by (c) NanoSight and fluorescence spectrophotometer, and (d) fluorescence correlation spectroscopy (FCS).

Table 2 The comparison of single-particle fluorescent brightness between PFBT nanoparticles and FITC-doped silica nanoparticles

\begin{tabular}{lll}
\hline Species & $\begin{array}{l}\text { Number-average } \\
\text { diameter }(\mathrm{nm})\end{array}$ & $\begin{array}{l}\text { Fluorescence } \\
\text { intensity slope }\end{array}$ \\
\hline PFBT nanoparticles & 183 & 12108 \\
FITC-doped silica nanoparticles & 200 & 432
\end{tabular}

\section{Conclusions}

In summary, PFBT nanoparticles with a size range of $50 \mathrm{~nm}$ to $200 \mathrm{~nm}$ were synthesized through amphiphilic polymer coprecipitation method, in order to have a systematic and thorough investigation into the size-dependent optical properties of CPNs in submicron range. It was demonstrated that the spectroscopic and fluorescent properties of CPNs changed little with the increase of size. The single-particle fluorescent brightness increased as CPNs grew larger. Furthermore, a quadratic dependence between single particle fluorescent brightness and the diameter of CPNs was discovered. Besides, the obtained CPNs in submicron range was much brighter than another kind of ultra-bright nanoparticles (FITC-doped silica spheres), verifying the strong fluorescence intensity of CPNs. This study provides important guidance for broader application of CPNs in biological detection.

\section{Conflicts of interest}

There are no conflicts to declare.

\section{Acknowledgements}

We acknowledge financial support for this work from The Key Research and Development Program of Zhejiang province (2017C03005) and SJTU funding (YG2015ZD11).

\section{References}

1 X. Michalet, F. F. Pinaud, L. A. Bentolila, J. M. Tsay, S. Doose, J. J. Li, G. Sundaresan, A. M. Wu, S. S. Gambhir and S. Weiss, Science, 2005, 307, 538-544.

2 J. C. G. Bunzli, Chem. Rev., 2010, 110, 2729-2755.

3 M. Bates, B. Huang, G. T. Dempsey and X. W. Zhuang, Science, 2007, 317, 1749-1753.

4 O. Tagit and N. Hildebrandt, ACS Sens., 2017, 2, 31-45.

5 E.-B. Cho, D. O. Volkov and I. Sokolov, Adv. Funct. Mater., 2011, 21, 3129-3135.

6 S. Bok, V. Korampally, C. M. Darr, W. R. Folk, L. Polo-Parada, K. Gangopadhyay and S. Gangopadhyay, Biosens. Bioelectron., 2013, 41, 409-416.

7 C. Grazon, J. Rieger, B. Charleux, G. Clavier and R. MéalletRenault, J. Phys. Chem. C, 2014, 118, 13945-13952. 
8 S. Bok, V. Korampally, L. Polo-Parada, V. Mamidi, G. A. Baker, K. Gangopadhyay, W. R. Folk, P. K. Dasgupta and S. Gangopadhyay, Nanotechnology, 2012, 23, 175601.

9 A. Ranzoni, A. den Hamer, T. Karoli, J. Buechler and M. A. Cooper, Anal. Chem., 2015, 87, 6150-6157.

10 C. Wu and D. T. Chiu, Angew. Chem., 2013, 52, 3086-3109.

11 C. Wu, T. Schneider, M. Zeigler, J. Yu, P. G. Schiro, D. R. Burnham, J. D. McNeill and D. T. Chiu, J. Am. Chem. Soc., 2010, 132, 15410-15417.

12 C. Wu, Y. Jin, T. Schneider, D. R. Burnham, P. B. Smith and D. T. Chiu, Angew. Chem., 2010, 49, 9436-9440.

13 Y. Rong, C. Wu, J. Yu, X. Zhang, F. Ye, M. Zeigler, M. E. Gallina, I. C. Wu, Y. Zhang, Y. H. Chan, W. Sun, K. Uvdal and D. T. Chiu, ACS Nano, 2013, 7, 376-384.

14 L. Xiong, F. Cao, X. Cao, Y. Guo, Y. Zhang and X. Cai, Bioconjugate Chem., 2015, 26, 817-821.

15 L. Xiong, A. J. Shuhendler and J. Rao, Nat. Commun., 2012, 3, 1193.

16 H. Y. Liu, P. J. Wu, S. Y. Kuo, C. P. Chen, E. H. Chang, C. Y. Wu and Y. H. Chan, J. Am. Chem. Soc., 2015, 137, 10420-10429.

17 C. Wu, S. J. Hansen, Q. Hou, J. Yu, M. Zeigler, Y. Jin, D. R. Burnham, J. D. McNeill, J. M. Olson and D. T. Chiu, Angew. Chem., 2011, 50, 3430-3434.

18 Y. Sun, W. Cao, S. Li, S. Jin, K. Hu, L. Hu, Y. Huang, X. Gao, Y. Wu and X. J. Liang, Sci. Rep., 2013, 3, 3036.

19 C. Cordovilla and T. M. Swager, J. Am. Chem. Soc., 2012, 134, 6932-6935.

20 X. Feng, F. Lv, L. Liu, H. Tang, C. Xing, Q. Yang and S. Wang, ACS Appl. Mater. Interfaces, 2010, 2, 2429-2435.

21 H. Shi, X. Ma, Q. Zhao, B. Liu, Q. Qu, Z. An, Y. Zhao and W. Huang, Adv. Funct. Mater., 2014, 24, 4823-4830.
22 M. C. Baier, J. Huber and S. Mecking, J. Am. Chem. Soc., 2009, 131, 14267-14273.

23 K. Li, J. Pan, S.-S. Feng, A. W. Wu, K.-Y. Pu, Y. Liu and B. Liu, Adv. Funct. Mater., 2009, 19, 3535-3542.

24 C. Szymanski, C. F. Wu, J. Hooper, M. A. Salazar, A. Perdomo, A. Dukes and J. McNeill, J. Phys. Chem. B, 2005, 109, 85438546.

25 B. Bao, Q. Fan, L. Wang and W. Huang, Sci. Sin.: Chim., 2014, 44, 282.

26 X. Zhang, J. Yu, C. Wu, Y. Jin, Y. Rang, F. Ye and D. T. Chiu, ACS Nano, 2012, 6, 5429-5439.

27 D. M. Rissin, D. H. Wilson and D. C. Duffy, The Immunoassay Handbook, 4th edn, 2013, pp. 223-242.

28 K. Sun, H. Chen, L. Wang, S. Yin, H. Wang, G. Xu, D. Chen, X. Zhang, C. Wu and W. Qin, ACS Appl. Mater. Interfaces, 2014, 6, 10802-10812.

29 D. Magde, E. Elson and W. W. Webb, Phys. Rev. Lett., 1972, 29, 705-708.

30 C. Dong and J. Ren, Analyst, 2010, 135, 1395-1399.

31 P. Zhang, L. Li, C. Dong, H. Qian and J. Ren, Anal. Chim. Acta, 2005, 546, 46-51.

32 N. Kurokawa, H. Yoshikawa, N. Hirota, K. Hyodo and H. Masuhara, ChemPhysChem, 2004, 5, 1609-1615.

33 J. K. Grey, D. Y. Kim, B. C. Norris, W. L. Miller and P. F. Barbara, J. Phys. Chem. B, 2006, 110, 25568-25572.

34 P. K. Jain, K. S. Lee, I. H. El-Sayed and M. A. El-Sayed, J. Phys. Chem. B, 2006, 110, 7238-7248.

35 J. Ries and P. Schwille, BioEssays, 2012, 34, 361-368.

36 Y. Zhu, H. Xu, K. Chen, J. Fu and H. Gu, Chem. Commun., 2014, 50, 14041-14044. 